\title{
Trombectomía ventricular izquierda por trombo apical extenso
}

José Picco*

Sebastián Wolff*

Emanuel González Dávila*

Gabriela García*

Pablo Burgos**

Claudio Burgos**

David Wolff*

* Servicio de Ecocardiografía Doppler. Hospital Italiano de Mendoza. Argentina

** Servicio de Cirugía Cardiovascular y Trasplante. Hospital Italiano de Mendoza. Argentina

Recibido: 25/05/2020

Aceptado: 18/10/2020

En línea: 31/12/2020

Citar como: Picco J, Wolff S, González Dávila E, García G, Burgos P, Burgos C, Wolff D. Trombectomía ventricular izquierda por trombo apical extenso. Rev Ecocar Pract (RETIC). 2020 (Dic); 3 (3): 10-12. doi: 10.37615/retic.v3n3a6.

Cite this as: Picco J, Wolff S, González Dávila E, García G, Burgos P, Burgos C, Wolff D. Left ventricular thrombectomy for extensive apical thrombus. Rev Ecocar Pract (RETIC). 2020 (Dic); 3 (3): 10-12. doi: 10.37615/retic.v3n3a6.

\section{Correspondencia}

José Picco piccojose@gmail.com

\begin{tabular}{l} 
Palabras clave \\
\hline$\triangleright$ Embolia \\
$\triangleright$ Trombo mural \\
$\triangleright$ Cirugía torácica \\
\end{tabular}

\section{Keywords}

\section{$\triangleright$ Embolism}

$\triangleright$ Mural thrombus

$\triangleright$ Thoracic surgery

\section{RESUMEN}

La presencia de trombos en cavidades izquierdas no suele ser algo que se observe con mucha frecuencia. Su presencia, en general, es secundaria a una agresión sobre el músculo cardíaco, como un infarto agudo de miocardio. Habitualmente, el manejo es conservador (mediante tratamiento anticoagulante), pero en el caso de trombos muy extensos o eventos embólicos se prefiere la vía quirúrgica.

ABSTRACT
The presence of thrombi in left cavities is not frequent. Its presence in general is secondary to a damage on the
heart muscle such as an acute myocardial infarction. Usually management is conservative (by anticoagulant
treatment), but in the case of very extensive thrombi or embolic events, the surgical route is preferred.

\section{Presentación del caso}

Varón de 40 años con factores de riesgo cardiovascular (fumador, sedentario, sobrepeso) y sin antecedentes patológicos cardiovasculares. En el contexto de un ingreso hospitalario por un cuadro respiratorio interpretado como neumonía adquirida en la comunidad asociado a signos de insuficiencia cardíaca, se realizó un ecocardiograma Doppler (Vídeo 1 y Vídeo 2) donde se observó un ventrículo izquierdo (VI) con hipertrofia excéntrica, hipoquinesia anteroseptal medial y apical, con aquinesia acentuada en el ápex y una imagen hiperecogénica adherida al ápex compatible con trombo mural. Se realizó tomografía computada (TC) con contraste como parte de su evaluación respiratoria y en busca de embolias. Se observaron múltiples imágenes compatibles con embolias en bazo, riñones e hígado. Una vez realizado el diagnóstico, el paciente fue reinterrogado y refirió haber padecido dolores precordiales autolimitados en las semanas previas a la internación, por los que no había consultado por miedo a contraer COVID-19.

Se evaluó la anatomía coronaria y se observó enfermedad severa de tres vasos. Con este cuadro, se decidió revascularización quirúrgica y extracción del trombo mural por ventriculotomía. Se realizaron puentes mamario de descendente anterior, venoso a circunfleja y venoso a coronaria derecha. Se extrajo el trombo mural mediante ventriculotomía izquierda y posterior cierre por planos (Vídeo 3 y Figura 1). La evolución postoperatoria fue buena (Vídeo 4), por lo que el paciente pudo ser dado de alta a los 7 días de la cirugía.

\section{Estudio por imagen}

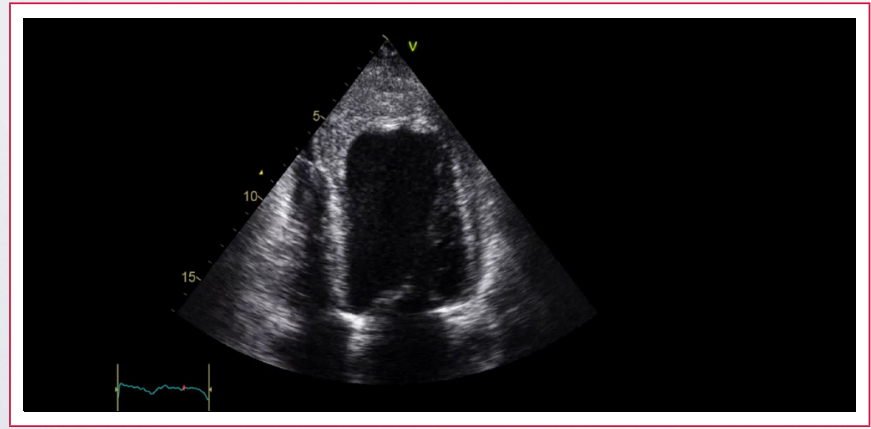

Vídeo 1. Vista apical de 4 cámaras centrada en el VI. Se observa extensa hipoquinesia septal y apical con imagen hiperecogénica adherida al ápex del VI 


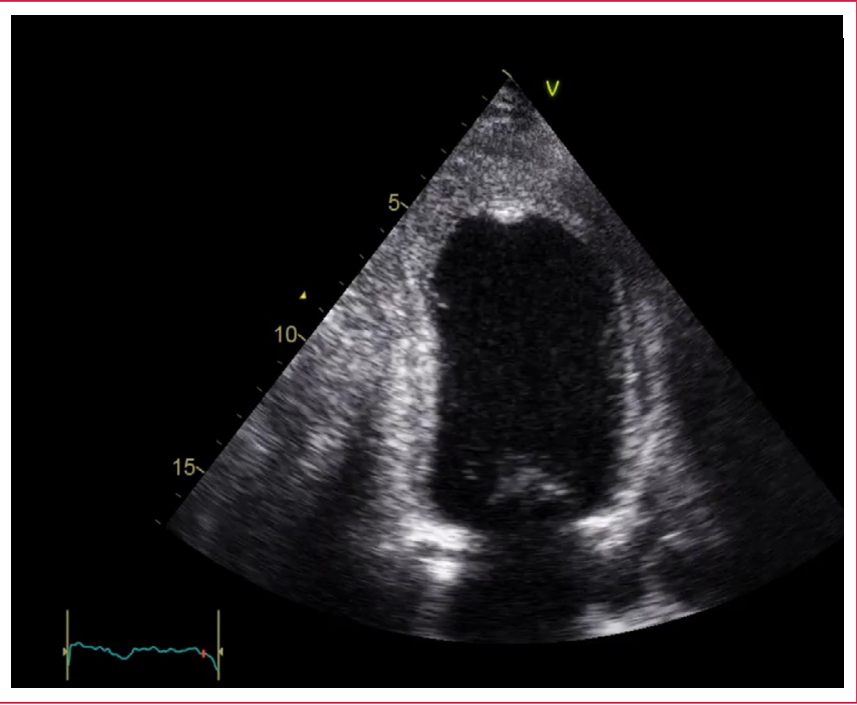

Vídeo 2. Vista apical de 2 cámaras centrada en el VI. Se observa, además de los trastornos de motilidad, la extensión del trombo mural

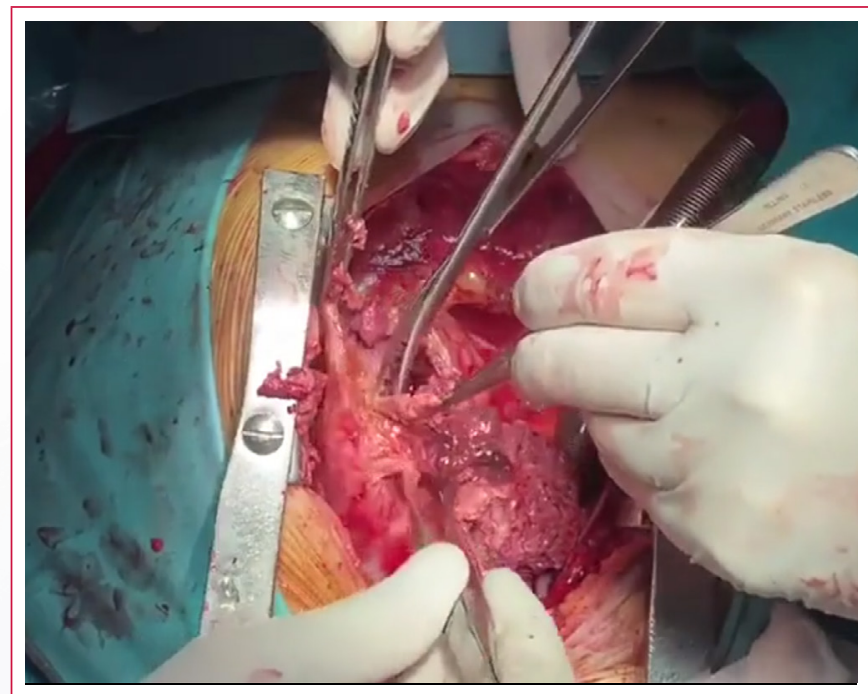

Vídeo 3. Extracción del trombo en quirófano a través de ventriculotomía izquierda

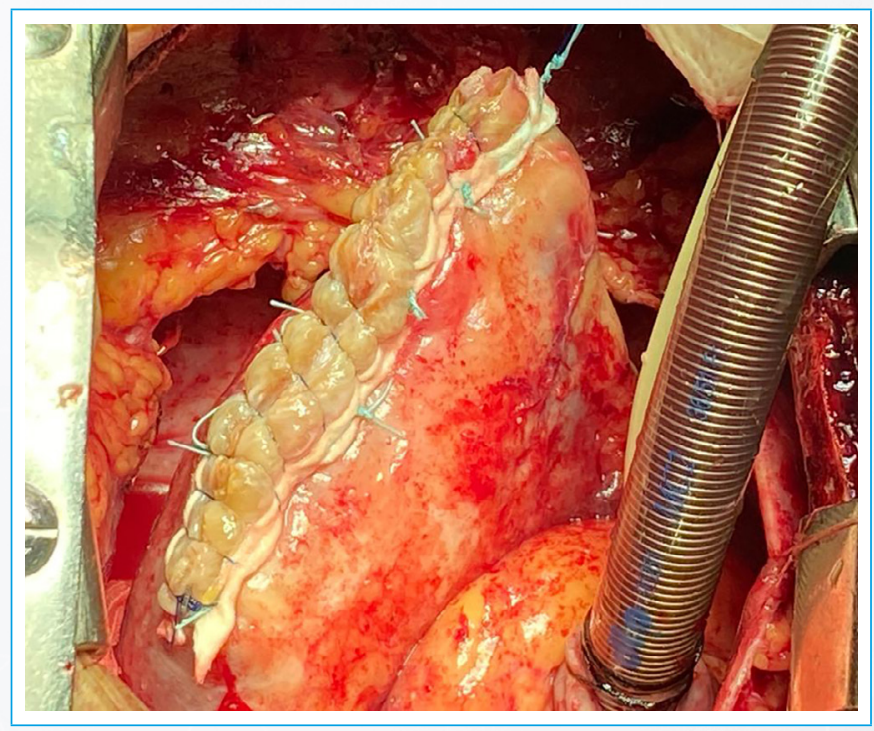

Figura 1. Vista del aspecto final de la sutura de cierre de la ventriculotomía

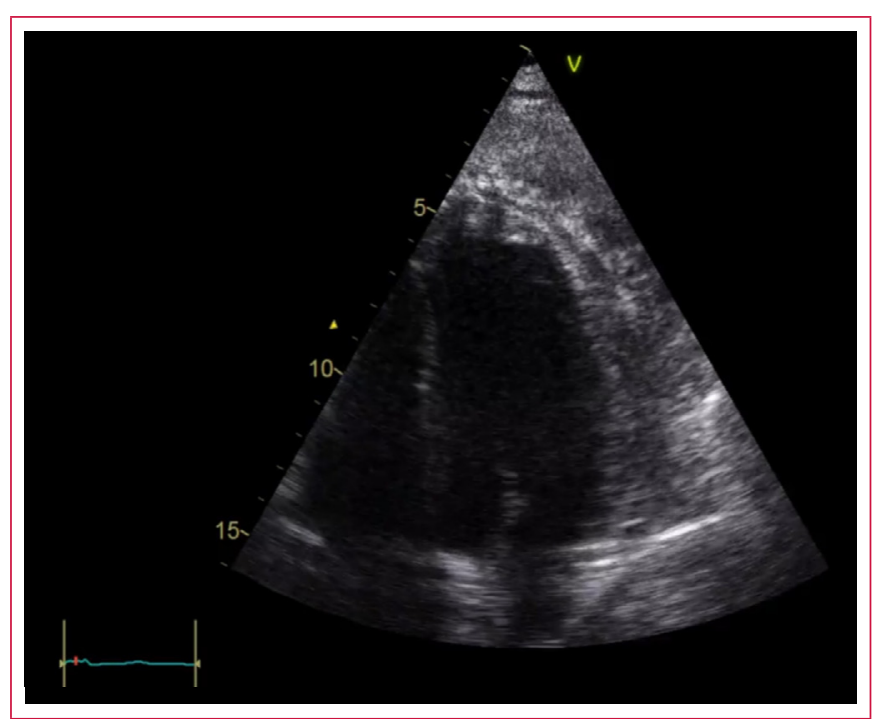

Vídeo 4. Vista apical de 4 cámaras posterior a la cirugía. Se puede observar la ausencia de trombo mural, si se compara con el Vídeo 1, y un deterioro moderado a severo de la función ventricular izquierda

\section{Discusión}

La presencia de un trombo en el VI es una complicación potencialmente mortal de la disfunción ventricular izquierda. Las opciones de tratamiento incluyen anticoagulación, trombólisis y trombectomía quirúrgica ${ }^{(1,2)}$. Los trombos pequeños e inmóviles pueden manejarse de forma segura con anticoagulación. El tratamiento para trombos móviles o muy grandes es controvertido ${ }^{(3)}$.

La primera opción de tratamiento para los trombos ventriculares es la anticoagulación; sin embargo, en el caso de un trombo extenso y con presencia de embolias múltiples, suele estar indicada la trombectomía quirúrgica urgente. El problema con la extirpación quirúrgica de un gran trombo del VI es la función ventricular, ya que a menudo se observan en pacientes con mala función en el VI. El enfoque convencional es la ventriculotomía izquierda ${ }^{(4,5)}$. Ésta proporciona visualización directa del trombo y permite eliminar por completo la masa adherida a la pared ventricular. Sin embargo, la ventriculotomía del VI puede agravar el deterioro de la función del $\mathrm{V}^{(6)}$, y debe evitarse en caso pacientes con deterioro severo de la función sistólica ventricular izquierda.

Otro posible enfoque para la extracción del trombo es mediante aortotomía, que ha sido reportada con toracoscopia videoasistida para facilitar la visualización ${ }^{(7)}$. El tamaño del trombo es a menudo el factor limitante en este enfoque. En nuestro caso reportado, el tamaño del trombo (como se observa en el Vídeo 3 ) era demasiado grande como para pasar a través de la válvula aórtica. Otro abordaje sugerido es el auricular izquierdo; éste no requiere incisión en el $\mathrm{VI}, \mathrm{y}$, por tanto, no afectaría la función ventricular. Este enfoque también proporciona una visualización adecuada del trombo y la extracción a través de la válvula mitral permite remover trombos de mayor tamaño que la vía transaórti$\mathrm{ca}^{(8-10)}$. La desventaja potencial del abordaje auricular izquierdo sería el espacio limitado para maniobrar, y debe reservarse sólo para trombos pediculados, por lo que tampoco habría sido apropiado en este paciente.

\section{Conclusión}

Presentamos el caso de un paciente con trombo ventricular apical extenso, posterior a un evento cardiovascular no tratado por falta de consulta, que 
evoluciona con insuficiencia cardíaca y embolias múltiples, resuelto mediante ventriculotomía con resultado favorable.

\section{Ideas para recordar}

- La trombectomía por ventriculotomía izquierda es una opción terapéutica para trombos muy grandes que están siendo fuente de embolias.

- Una posible secuela de cualquier ventriculotomía es el deterioro de la función ventricular.

\section{Bibliografía}

1. Lee JM, Park JJ, Jung HW, Cho YS, Oh IY, Yoon CH, et al. Left ventricular thrombus and subsequent thromboembolism, comparison of anticoagulation, surgical removal, and antiplatelet agents. J Atheroscler Thromb 2013; 20: 73-93.

2. Leick J, Szardien S, Liebetrau C, Willmer M, Fischer-Rasokat U, Kempfert J, et al. Mobile left ventricular thrombus in left ventricular dysfunction: case report and review of literature. Clin Res Cardio/ 2013; 102: 479-484.
3. Stratton JR, Lighty GW, Pearlman AS, Ritchie JL. Detection of left ventricular thrombus by two-dimensional echocardiography: sensitivity, specificity, and causes of uncertainty. Circulation 1982; 66: 156-166.

4. Yadava OP, Yadav S, Juneja S, Chopra VK, Passey R, Ghadiok R. Left ventricular thrombus sans overt cardiac pathology. Ann Thorac Surg 2003; 76: 623-625.

5. Suzuki R, Kudo T, Kurazumi H, Takahashi M, Shirasawa B, Mikamo A, et al. Transapical extirpation of a left ventricular thrombus in Takotsubo cardiomyopathy. J Cardiothorac Surg 2013; 8: 135.

6. DiBernardo LR, Kirshbom PM, Skaryak LA, Quarterman RL, Johnson RL, Davies MJ, et al. Acute functional consequences of left ventriculotomy. Ann Thorac Surg 1998; 66: 159-165.

7. Tsukube T, Okada M, Ootaki Y, Tsuji Y, Yamashita C. Transaortic video-assisted removal of a left ventricular thrombus. Ann Thorac Surg 1999; 68: 1063-1065.

8. Kuh JH, Seo Y. Transatrial resection of a left ventricular thrombus after acute myocarditis. Heart Vessels 2005; 20: 230-232.

9. Lutz CJ, Bhamidipati CM, Ford B, Swartz M, Hauser M, Kyobe M, et al. Robotic-assisted excision of a left ventricular thrombus. Innovations (Phila) 2007; 2: $251-253$

10. Early GL, Ballenger M, Hannah H, Roberts SR. Simplified method of left ventricular thrombectomy. Ann Thorac Surg 2001; 72: 953-954 